$\xi=-1$

\title{
An Inventory Model with Embeeded Acceptance Sampling Plan
}

\author{
N. Konda Reddy ${ }^{1 *}$, K. Murali Krishna ${ }^{2}$, A.V.N. Murty ${ }^{3}$ \\ ${ }^{1}$ Department of Mathematics, Koneru Lakshmaiah Education Foundation, Greenfields, Vaddeswaram, Guntur Dist, A.P, INDIA. \\ ${ }^{2}$ Research Scholar, Department of Mathematics, Koneru Lakshmaiah Education Foundation, Greenfields, Vaddeswaram, Guntur Dist, \\ A.P,INDIA. \\ ${ }^{3}$ Department of Management, Koneru Lakshmaiah Education Foundation, Greenfields, Vaddeswaram, Guntur Dist, A.P, INDIA. \\ *Corresponding author E-mail: nkrstats@kluniversity.in
}

\begin{abstract}
The classical inventory model assumes that the quality of the items received matches with the quality required. In many cases the deviation in quality results in additional costs to the stockiest and sometimes lead to rejection of the received lot. The cost of inspection along with the other inventory costs can be unified into a single model by embedding an acceptance sampling plan into the inventory model. When the lot is accepted there is a possibility of unseen defectives, which may reach the customer leading to loss of goodwill. If the lot is rejected on inspection, the model suggests rectification and removal of non-confirming units. In this paper a model is developed to determine (i) the Economic Order Quantity and (ii) a single sample plan of the attribute type, which minimizes the Average Total Inspection. The model utilizes spreadsheet solutions to handle statistical functions.
\end{abstract}

Keywords: ATI; Excel; Inventory; Sampling Plan;

\section{Introduction}

\subsection{The Nature of Inventory Problems}

Inventory control is one of the fundamental areas of management in any organization. According to Love (1979), the term inventory refers to "an idle resource of any kind having an economic value". Inventory of equipment, spare parts, domestic consumables etc., are some familiar words in which the word inventory is understood as 'physical stock awaiting its use or consumption'.

The very existence of inventory is due to a temporary halt between the supply and demand process for the commodity under consideration. It is normally not possible to get items as and when they are demanded. Certain amount of inventory is always necessary for the smooth running of the business. However, neither a high inventory nor a low inventory is desirable because there are costs associated with keeping stock.

A scientific approach to inventory management is therefore necessary for effective maintenance of the production / business.

\subsection{The Behavior of Demand, Supply and Their Math- ematical Treatment}

An inventory system basically contains an input process and an output process, which refer to supply and demand aspects respectively. The supply would be made either by purchasing the item from external resources or by producing the same. In case of production, the supply rate is finite and the way inventory is built, is called finite replenishment process. When the quantity ordered is supplied entirely in a single shipment, the method is called infinite rate of replenishment. Usually there will be a time lag between placement of an order and the actual receipt of the order quantity. This is called lead time and is generally denoted by L, which may be either a known constant or a random variable, with some known distribution.

Statistical procedures are normally adopted by managers to estimate the demand for a period and buffer stocks are maintenance to reduce the risk of facing a shortage. But a common question is "at what cost can we avert a shortage?". To answer this question we have to use a 'model' that explains the economic aspects of inventory. Such a model shall be analysed and an inventory ordering rule should be arrived at.

When the demand is assumed to be a known constant or a variable with variability over time period, it is called deterministic demand. Demand happens to be deterministic when the customers book their orders with the stockist. A dynamic, known demand situation contains $D_{i}$ units of demand for the $i^{\text {th }}$ period, $i=1,2 \ldots N$. Sometimes, the quantity demanded cannot be predicted with certainty but can be described by a probabilistic distribution in a specified interval. Statistical tools can be used in the study of such demand. In some other cases demand is unknown. It is estimated with the help of the demand pattern for a known correlated product.

The inventory level of a commodity at time $t$ is denoted by $I(t)$ and it is a fundamental variable for the mathematical study of the inventory system. Let $\mathrm{I}(0)$ be the initial inventory, $\mathrm{S}(\mathrm{u})$ and $\mathrm{D}(\mathrm{u})$ denote respectively the supply and demand at any time $\mathrm{u}, 0 \leq \mathrm{u} \leq$ t. Then $I(t)$ can be expressed as

$$
\mathrm{I}(\mathrm{t})=\mathrm{I}(0)+\int_{0}^{\mathrm{t}}\{\mathrm{S}(\mathrm{u})-\mathrm{D}(\mathrm{u})\} \mathrm{du}
$$

$\mathrm{I}(\mathrm{t})$ could be positive or negative and for a known T, the inventory held during $(0, \mathrm{~T})$ becomes

$$
\int_{0}^{\mathrm{T}} \mathrm{I}(\mathrm{t}) \mathrm{dt}
$$


Negative inventory is simply a shortage.

The study of $\mathrm{I}(\mathrm{t})$ and its behavior accounts for proper modeling of inventory costs.

\subsection{Various Costs Affecting Inventory Control}

There are various costs associated with inventory maintenance. The main among them are as follows.

Holding cost (h)

This is the cost associated with holding one unit of inventory for one unit of time. This is usually expressed as a fraction of rupee per unit per unit time and denoted by $h$. This cost arises due to the payments made towards inventory maintenance in the form of taxes, insurance, protection against pilferage, deterioration, damage and cost of storage space. Another important factor of holding cost is the opportunity loss associated with the money, tied up in the form of inventory.

The common method of modeling inventory holding cost is to assume that it is proportional to the average inventory held during the period. Let $\mathrm{T}$ denote the planning period during which the inventory is held. Then the average inventory during a period $(0, \mathrm{~T})$ is given by

$$
\overline{\mathrm{I}}=\frac{\left[\int_{0}^{\mathrm{T}} \mathrm{I}(\mathrm{t}) \mathrm{dt}\right]}{\mathrm{T}}
$$

The average holding cost during the period is simply $\mathrm{h} \overline{\mathrm{I}}$. If $\mathrm{CP}$ denote cost price of the item excluding the cost of capital, then the holding cost per unit can be written as $\mathrm{H}=\mathrm{iCP}$, where $\mathrm{i}$ denotes the rate of interest or cost of capital.

\section{Shortage cost/Stock-out cost $(\boldsymbol{\pi})$}

This is the cost of unsatisfied demand. When the item is temporarily out of stock and a demand occurs, the customer may either cancel the order or wait until the arrival of stock. When the customer cancels an order, the profit that could accrue out of it, is lost and in addition it may result in a loss of good will to the management. This cost is denoted by $\pi$ per unit time.

When the customer is willing to wait until stock arrives for the inventory, the demands is said to be backordered or backlogged.

This is denoted by $\hat{\pi}$ per unit time. The shortage cost should be proportional to the quantity short or proportional to both quantity short and time.

Set up cost/Ordering cost/Replenishment cost (A)

This is denoted by A per production run or per purchase order The cost is assumed to be independent of the quantity ordered or produced. It is a fixed cost and it is worked out basing on the number of orders placed in a time unit (like one year) and the expenditure involved. In some cases the ordering cost could also include a variable component like $\mathrm{A}=\mathrm{A}^{\prime}+\mathrm{bQ}$, where $\mathrm{b}$ is a positive constant that represents a component like material handling charges (loading/ unloading). $\mathrm{A}^{\prime}$ is a fixed component independent of the lot size.

\section{Material cost}

The cost of material is less affected by the decision regarding inventory maintenance in many cases. However with quantity discounts or price fluctuations, the material cost becomes one of the variable costs of the system. When the price of the item is subject to inflation, a hike in price may be expected for future purchases. In such cases, a significant saving on the material cost can be realised by purchasing extra quantity before the price goes up. This situation arises when there are inflationary conditions Buzacolt (1975) and Sambandam (1984) have derived the EOQ for this type of situation.

\section{Operating costs/System control costs}

These are costs associated with data processing, forecasting, stock reviewing and placing timely orders, check up the material flow in the system etc., which are essential for implementing any invento- ry policy. According to Silver (1981), a major portion of literature on inventory models does not seem to have given due consideration to these costs.

The estimation of cost parameters for any inventory system is one of the important phases of inventory decision making. These costs are to be estimated from the management accounts and the method of determination is discussed by Love (1979) and Lambert et al (1967).

\subsection{Sampling Inspection Methods}

The term quality is understood as fitness for use. Several statistical tools are used by the manufacturer to assure quality of the manufactured product. These are two major areas in quality assurance, namely product control and process control.

Quality assurance of incoming material (from vendors) and outgoing products belong to the category of product control. A lot of theoretical research as well as practical procedures have been provided by Duncan (1952), Schilling (1982), Dodge and Roming (1959) and other researchers. Montgomery (1977) contains several details about acceptance sampling.

Once the material is taken in for production, a lot of control is exercised to assure the desired quality of the final product. This branch of Statistical Quality Control is called process control. It includes sampling inspection from the process estimation of product quality, testing of hypotheses about the process etc.

Acceptance sampling is a methodology meant for sentencing a lot, basing on a sample from it. The quality assessment of the lot is based on either an attribute like colour, taste or a measured characteristic (variable) like length, weight etc.

There are several sampling plans in use as mentioned below.

Single Sampling Plan (SSP)

Rectifying Inspection Plans

Double Sampling Plan (DSP)

Multiple Sampling Plan (MSP)

Continuous Sampling Plan (CSP)

Skip lot Sampling Plan (SkSP)

Chain Sampling Plan (ChSP)

A single sampling plan is defined by the parameters, $\mathrm{N}, \mathrm{n}$ and $\mathrm{c}$, where $\mathrm{N}$ denotes lot size, $\mathrm{n}$ denotes sample size and $\mathrm{c}$ denotes critical number, for lot rejection.

Some basic parameters for designing a sampling plan are as follows.

Acceptable Quality Level (AQL)

Lot Tolerance Percent Defective (LTPD)

Producer's risk $(\alpha)$

Consumer's risk $(\beta)$

\subsection{The Single Sampling Plan Operates as Follows}

From the lot we take a random sample of size ' $n$ ' and note the number of defectives ' $\mathrm{d}$ '. If $\mathrm{d} \geq \mathrm{c}$ we reject the lot; otherwise accept. Obviously there could be errors in judging the lot quality, because the inspection is based on the sampling. Using statistical testing of hypothesis, we fix $\alpha$ say at 10 percent and determine the values $n$ and $c$ such that the $\beta$ risk is minimized.

If a lot is rejected, a practice in production system is to "rectify" the lot. This is done with 100 percent inspection of the remaining portion lot (screening). The defectives are replaced with good units or they are simply removed leading to a reduced lot.

When a lot is rejected, rectifying inspection ensures zero defectives. If the lot is accepted, certain portion of defectives goes unnoticed and reached the customer. The percentage of such defectives is called Average Outgoing Quality (AOQ).

While it is not a difficult job to design a sampling plan, certain predetermined plans, primarily meant for military application have been prepared by Dodge and his team. Examples of such sampling plans are MILSTD-105D and Dodge-Romig plans. 
The designing of a sampling plan can be made as a part of determining the lot size. The inventory model then includes cost components arising out of sampling and testing, the cost of rework, cost of defectives reaching the customers and so on.

\subsection{Review of Literature}

Inventory models with quality aspects are commonly studied in a production environment. Quality aspects cannot be ignored in purchased items also. It is a common observation that in case of several consumer goods, a high level of inventory does not mean a high sales rate. Customers may reject some of the items kept the shelf if they do not confirm to their expectations on quality. As a result, the stockiest has to assure the quality of the material before putting it for sale/consumption.

Silver (1976), Wie Shih (1980) and Kalro and Gohil(1982) are some basic references in this direction. Sometimes the quantity received against the order will be uncertain due to quality problems while in some cases the supplier makes an excess-supply than what is asked, in order to make up for the possible defectives. When it is found, after a sample testing, that the quality is not up to the mark, the stockist may find the lot acceptable but not at the quoted price. He then claims a price-discount as a sort of compensation for the poor quality. During usage, he would make-up for quality by putting extra quantity to obtain the desired output The interesting aspect of this type of sales is that the demand distribution drifts to the right or left of its central value. Sarma and Mohan Naidu (1997) have worked on an inventory problem with this type of drifted demand. A similar situation occurs in sales context, when the stockist offers extra quantity for the same price possibly to cover up a lower quality. When the customers compares between price and quality, this type of incentives often work well.

In this paper we study a simple lot size inventory model governed by deterministic demand. We adopt a single sampling plan of the attribute type and determine the costs arising out of inspection and the penalty cost due to bad items reaching the customer by a slip of inspection. We determine the EOQ for this situation and illustrate the model with the help of Excel worksheet. The model is numerically illustrated and the sensitivity is examined with respect to critical parameters.

\section{Problem Environment and Mathematical Model}

Consider an inventory problem controlled by the EOQ policy with deterministically known demand of $\mathrm{D}$ units/unit time. When an order is placed there is a fixed cost of A and the unit holding cost is $\mathrm{h}$ per unit/unit time and the lead time is not significant. The received lot is subjected to acceptance sampling using a single sampling plan. A sample of $\mathrm{n}$ units are inspected at random from the lot and the lot is accepted if the number of defectives in the sample is lee than or equal to c. The lots from the supplier contain a a known fraction defective $p$. The inspection is assumed to be error free and the probability of accepting a lot is $\mathrm{P}_{\mathrm{a}}$.

When the lot is accepted, the defectives found in the sample can't be replaced immediately and they are kept aside for replacement by the supplier or they are taken back by the supplier at no extra cost at the time of next delivery. As a result of the un-inspected portion of every accepted lot contains $\mathrm{p}(\mathrm{Q}-\mathrm{n})$ units on an average. These defectives may reach the customer during sales. If a defective reaches the customer, there is a penalty cost of $v$ per unit.

Then the expected number of defectives that would the reach the customer becomes $\quad v p \mathrm{~Pa}(\mathrm{Q}-n p)$ and this increases linearly with Q. When the lot is rejected after sampling inspection, $100 \%$ inspection is carried out by inspecting the remaining (Q-n) units This is the conventional concept of rectifying inspection, which is better applicable for a production system in which there is a provi- sion for replacement of defective items with good items. However in the case of purchased items from a supplier, the defectives cannot be replaced, because good items for replacement for are not immediately available. Sekhar, Sarma and Goyal (1995) have studied a situation of this type, where rectification leads to a reduced lot size in a two stage production system. In the present case the rectification result in a reduced lot size of $Q^{2}$ units, where $Q^{\mathrm{E}} \leq \mathrm{Q}$.

Consider the following proportion.

Proposition-1: The expected lot size available for for sales/consumption is $Q^{2}=p \mathrm{~Pa}(\mathrm{Q}-n p)+\mathrm{Q}(1-\mathrm{P})$ and $Q^{\mathrm{e}} \leq \mathrm{Q}$. The equality holds when all the defectives are replaced by good items.

Proof: The result follows by noting that that every accepted lot contains on an average (Q-n)p good items and this happens with probability $\mathrm{P}_{\mathrm{a}}$. The rejected lot contains $\mathrm{Q}(1-\mathrm{p})$ good items.

$Q^{\prime \prime}=p \operatorname{Pa}(\mathrm{Q}-n p)+\mathrm{Q}(1-\mathrm{P})$

Hence the proposition.

The expression given in (1) determines the expected cycle length $\mathrm{E}(\mathrm{t})=\frac{Q}{D}$. While using it with the cost function this form leads to a complicated expression to determine Q. The usual cycle length is $\frac{Q}{D}$ when there are no defectives. In the present case defectives are remover either during the inspection or during usage. The ultimate expected cost that will be hold Q(1-p). Hence the cycle length can be taken as $\mathrm{E}(\mathrm{t})=\frac{Q(1-p)}{D}$

The following cost components are related to the lot size.

The fraction defective that reaches the customer from the accepted lot is simply the Average Outgoing Quality (AOQ) given by $p=$ $p \mathrm{~Pa}(\mathrm{Q}-n)$. The expected number of defectives that reaches the customer will be $p Q$ and cost due to these defectives is $v p \mathrm{~Pa}(\mathrm{Q}$ $n p)$.

The other cost relating to sampling plan is the cost of inspection. The average number of items inspected either in sampling or in screening is called the Average Total Inspection is given by ATI = $n+(\mathrm{Q}-n)\left(1-\mathrm{P}_{\mathrm{a}}\right)$. If the inspection cost $g$ per unit, the cost of inspection in any cycle will be $\mathrm{g}\left[n+(\mathrm{Q}-n)\left(1-\mathrm{P}_{\mathrm{a}}\right)\right.$.

Now the sum of the cost in each cycle becomes

$\mathrm{K}(\mathrm{t}, \mathrm{Q})=\left(1-\mathrm{P}_{\mathrm{a}}\right)\left[A+\frac{h Q(1-p) t}{2}+\mathrm{g} Q\right]+\mathrm{P}_{\mathrm{a}}$
$\left[A+\frac{h(Q-n) p t}{2}+\mathrm{g} n+v p(Q-n)\right]$

The total cost per unit time is then given by

$\mathrm{K}(\mathrm{Q})=$

$\frac{D}{Q}\left[A+\frac{h Q^{r}}{2} \cdot \frac{Q^{r}}{D}+\mathrm{g} Q\left(1-P_{a}+\mathrm{gn} P_{a}+v p P_{a}(Q-\right.\right.$

n)]

Using the relation $\frac{Q}{Q}=\frac{Q}{Q(1-P)}=\frac{1}{(1-P)}$ and simplifying (2) becomes

$\mathrm{K}(\mathrm{Q})=\frac{D}{Q^{\prime}}\left[A+g n P_{a}+v p P_{a}(Q-n)\right]+$

$\frac{h Q^{\prime}}{2}+\frac{D}{(1-p)}\left[g\left(1-P_{a}\right)\right]$

$=\frac{D}{Q(1-p)}\left[A+n P_{a}(\mathrm{~g}-v p)\right]+$

$\frac{h Q^{r}}{2}+\frac{D}{(1-p)}\left[g\left(1-P_{a}\right)+v p P_{a}\right]$

It can be seen from the proposition below that $K(Q)$ is convex in Q.

Proposition-2: $\mathrm{K}(\mathrm{Q})$ is convex in $\mathrm{Q}$ for $\mathrm{g} \geq p v$. 
The result follows from $\mathrm{K}(\mathrm{Q})$ by noting that the second derivative of $\mathrm{K}(\mathrm{Q})$ with respect to $\mathrm{Q}$ is $\frac{2 D\left[A+n P_{a}(\mathrm{~g}-v p)\right]}{Q^{\mathrm{g}}(1-p)}$

which is positive only when $\mathrm{g} \geq p v$.

Hence the proof.

The value of $Q$ that minimizes $K(Q)$ is the solution of $\frac{\mathrm{dK}(\mathrm{Q})}{\mathrm{dQ}}=0$ and is given by

$Q^{*}=\sqrt{\frac{2 D\left[A+n P_{a}(\mathrm{~g}-v p)\right]}{h(1-p)^{2}}}$

This formula resembles that of the classical EOQ formula with setup/ordering cost $\mathrm{A}^{*}$

where $A^{*}=\left[A+n P_{a}\left(g-v p^{2}\right)\right]$.

The corresponding minimum cost would be $\mathrm{K}\left(Q^{*}\right)=\sqrt{\left(2 D\left[A+n P_{a}(g-v p)\right]\left\{h(1-p)^{2}\right\}\right]}$.

The model result agrees with the classical EOQ model when $n=0$ and $p=0$. When $n=0$ and $p>0$, the value of $\mathrm{Q}^{*}$ agrees with that of Wie Shih (1980).

\subsection{Evaluation of the Model}

The value of $\mathrm{Q}^{*}$ depends on the value of $\mathrm{P}_{\mathrm{a}}$ but $n$ and $\mathrm{P}_{\mathrm{a}}$ is not known initially. It depends on the sampling plan adopted. One method of determining the plan parameters is to use a Type-B OC curve with two known points $\{\mathrm{AQL},(1-\alpha)\}$ and $\{\mathrm{LTPD}, \beta\}$. Another procedure is derive a plan that minimizes the ATI for the given level of LTPD. It can be seen (Montgomery (1980)) that the values of $c$ and $n$ uniquely determine the ATI. We can also use a standard sampling plan like the one suggested by Dodge and Romig (1947). We now discuss the ATI method and illustrate the working of the model.

\subsection{Method based on Average Total Inspected}

This method requires the calculation of $\mathrm{P}_{\mathrm{a}}$ for a given level of the incoming quality $p$. We use Type-B OC with Poisson approximation to find $\mathrm{P}_{\mathrm{a}}$.

The following stepwise method helps in determining Q

Step-1: Put $n=0$ and get $\mathrm{Q}_{0}=\sqrt{\frac{2 A D}{h(1-p)^{2}}}$

Step-2: Given LTPD, $\alpha$ and $\beta$, determine the plan that minimizes ATI taking the lot size as $\mathrm{Q}_{0}$

Step-3: Note the values of $c, n, \mathrm{P}_{\mathrm{a}}$ and ATI

Step-4: Using the values of $n$ and $\mathrm{P}_{\mathrm{a}}$ obtained in step-1 get a new value of $\mathrm{Q}$ say $\mathrm{Q}_{1}$

Step-5: Repeat the method until two consecutive values of $\mathrm{Q}$ or ATI become close to each. This gives the optimal Q*

The Excel worksheet has a built in function to calculate the cumulative Poisson probability. This has two steps, one for computing ATI and the other for finding the EOQ. Consider the following illustration.

\section{Illustration-1}

Consider the parameters $\mathrm{D}=10000, \mathrm{~A}=150, h=3, g=5, v=15$, $p=0.02$. The trial value of $\mathrm{Q}$ is found to be $\mathrm{Q}_{0}=1020$ units. With these values the trail value of $\mathrm{Q}_{0}$ with $\mathrm{n}=0$ is found to be 1020 units. Taking this as the lot size the sampling plan at a given LTPD of 0.10 is found by minimizing ATI. The calculations were performed using Excel work sheet and the results are shown in table-1. The Excel paste function Poisson (x, mean, cumulative) has been used to work out the type-B OC curve.
Table-1: Determination of Sampling that minimizes ATI for LTPD $=10 \%$

\begin{tabular}{|l|l|l|l|l|l|}
\hline $\mathbf{C}$ & $n$ & $\lambda$ & $\mathbf{P}_{\mathbf{a}}$ & $\left(\mathbf{1 - P _ { \mathbf { a } }}\right)$ & $\mathbf{A T I}$ \\
\hline 0 & 29 & 0.58 & 0.5599 & 0.4401 & 465.14 \\
\hline 1 & 49 & 0.98 & 0.7431 & 0.2569 & 298.43 \\
\hline 2 & 67 & 1.34 & 0.8478 & 0.1522 & 212.04 \\
\hline 3 & 84 & 1.68 & 0.9098 & 0.0902 & 168.45 \\
\hline 4 & 100 & 2 & 0.9473 & 0.0527 & 148.44 \\
\hline $\mathbf{5}$ & $\mathbf{1 1 6}$ & $\mathbf{2 . 3 2}$ & $\mathbf{0 . 9 6 8 9}$ & $\mathbf{0 . 0 3 1 1}$ & $\mathbf{1 4 4 . 0 8}$ \\
\hline 6 & 132 & 2.64 & 0.9815 & 0.0185 & 148.41 \\
\hline 7 & 148 & 2.96 & 0.9889 & 0.0111 & 157.65 \\
\hline 8 & 164 & 3.28 & 0.9933 & 0.0067 & 169.70 \\
\hline 9 & 180 & 3.6 & 0.9960 & 0.0040 & 183.38 \\
\hline 10 & 196 & 3.92 & 0.9976 & 0.0024 & 198.01 \\
\hline 11 & 212 & 4.24 & 0.9985 & 0.0015 & 213.20 \\
\hline 12 & 228 & 4.56 & 0.9991 & 0.0009 & 228.72 \\
\hline 13 & 244 & 4.88 & 0.9994 & 0.0006 & 244.43 \\
\hline
\end{tabular}

The minimum of ATI is 144 has been obtained at $n=116$ and $c=$ 5. The probability of accepting the lot is $P_{a}=0.9689$. Using this new value of $Q$ is found to be 1695 . Repeat this procedure a better value of $\mathrm{Q}$ is found to be 1776 and no further improvement could be found in the ATI with this value. The iterations are shown in table- 2 .

Table-2: Calculation of EOQ with a given sampling plan

\begin{tabular}{|l|l|l|l|l|}
\hline Trial & $\mathrm{N}$ & $\mathrm{C}$ & $\mathrm{Pa}$ & $\mathrm{Q}^{*}$ \\
\hline 1 & 0 & 0 & 0 & 980 \\
\hline 2 & 116 & 5 & 0.9689 & 2085 \\
\hline 3 & 132 & 6 & 0.9815 & 2205 \\
\hline 4 & 132 & 6 & 0.9815 & 2205 \\
\hline
\end{tabular}

\subsection{Sensitivity of the Model to Changes in the Sampling Plan}

The sampling plan and the EOQ model are influenced by the values of $p, n$ and $c$. Let us use the parameters given in illustration-1. We consider three single sampling plans each with $n=50$ and take $c=1,2$ and 3 for comparison. For different values of $p$ the corresponding EOQ and the minimum cost are evaluated. The probability of accepting the lot is calculated using Poisson function and the results are shown in table- 3 .

Table-3: Sensitivity of the model to changes in $p$ and $c$

\begin{tabular}{|l|l|l|l|l|l|l|}
\hline \multirow{2}{*}{$\mathrm{P} \quad \mathrm{p}=1$} & \multicolumn{3}{|l|}{$\mathrm{c}=2$} & $\mathrm{c}=2$ \\
\cline { 2 - 7 } & EOQ & COST & EOQ & COST & EOQ & COST \\
\hline 0.00 & 2121.49 & 3996.76 & 2150.76 & 4129.80 & 2155.60 & 4151.56 \\
\hline 0.01 & 2049.44 & 3600.47 & 2121.38 & 3931.19 & 2144.83 & 4035.41 \\
\hline 0.02 & 1974.63 & 3188.15 & 2074.56 & 3659.00 & 2122.76 & 3873.01 \\
\hline 0.03 & 1909.48 & 2817.64 & 2019.13 & 3351.40 & 2089.03 & 3664.29 \\
\hline 0.04 & 1857.68 & 2511.15 & 1963.07 & 3042.63 & 2046.75 & 3423.06 \\
\hline 0.05 & 1818.80 & 2272.53 & 1911.69 & 2756.69 & 2000.27 & 3167.70 \\
\hline 0.06 & 1790.77 & 2095.88 & 1867.74 & 2507.67 & 1953.70 & 2915.47 \\
\hline 0.07 & 1771.17 & 1970.69 & 1832.03 & 2301.62 & 1910.16 & 2680.03 \\
\hline 0.08 & 1757.77 & 1885.21 & 1804.17 & 2138.68 & 1871.61 & 2470.59 \\
\hline 0.09 & 1748.78 & 1828.62 & 1783.13 & 2014.99 & 1838.95 & 2292.17 \\
\hline 0.10 & 1742.83 & 1792.06 & 1767.65 & 1924.46 & 1812.28 & 2146.08 \\
\hline
\end{tabular}

It follows from the above analysis that as the fraction defective of lots increases, the EOQ decreases while the minimum cost increases.

\section{Conclusion}

The effect of using sampling plans in purchase inventory problem is highlighted in this paper. The lot fraction defective is shown to have significant influence on the lot size of inventory. It is observed that when the lots arrive from the vendor with poor quality, the lot size should be reduced because it increases cost of inspection and also the penalty due to sale of defective items. This mod- 
el has application to several consumer goods in which the user wishes to know the quality of the items before paying for it.

\section{Acknowledgement}

The authors are very thanks to the reviewers and editors for valuable comments, remarks and suggestions for improving the content of the paper.

\section{References}

[1] Montgomery, D.C, An Introduction to Statistical Quality Control, John \& Wiley, Inc, (1977)

[2] Wei Shih, Optimal Inventory Policies when Stock Result from Defective Products, International Journal of Production Research, Vol.8 (1980), pp. 677-686.

[3] Karlo, A.H and Gohil, M.M, A lot Size Model with Backlogging when the Amount Received is Uncertain, International Journal of Production Research, Vol.20 No. 6, (1982), pp.775-786.

[4] Silver, E.A and Peterson, R, Decision systems for inventory management and production planning, Wiley, $2^{\text {nd }}$ Edition (1985), New Delhi.

[5] Sekhar Naidu, B.M.C, Sarma, K.V.S. and Goyal, S.K, Optimal Two Stage Production-Inventory Policy with Whole lot and Split lots, Production Planning and Control, Vol.6, No. 2 (1995)

[6] Mohan Naidu, G and Sarma, K.V.S, Optimal order level systems for products with imperfect quality, Industrial Engineering Journal, Vol.24, No.5 (1998) 